
\title{
28 Research Square \\ Fn14 Exacerbates Acute Lung Injury by Activating the NLRP3 Inflammasome in Mice
}

\section{Xin-Xin Guan}

Central South University

Hui-Hui Yang

Central South University

Wen-Jing Zhong

Central South University

Jia-Xi Duan

Central South University

Chen-Yu Zhang

Central South University

Hui-Ling Jiang

Central South University

Yang Xiang

Central South University

Yong Zhou

Central South University

Cha-Xiang Guan ( $\sim$ guanchaxiang@csu.edu.cn )

Central South University

\section{Research Article}

Keywords: acute lung injury, Fn14, NLRP3 inflammasome, TWEAK, macrophage

Posted Date: November 2nd, 2021

DOI: https://doi.org/10.21203/rs.3.rs-1005882/v1

License: (c) (i) This work is licensed under a Creative Commons Attribution 4.0 International License.

Read Full License 


\section{Abstract}

Background: Uncontrolled inflammation is an important factor in the occurrence and development of acute lung injury (ALI). Fibroblast growth factor-inducible 14 (Fn14) takes part in the pathological process of a variety of inflammatory diseases. However, the role of Fn14 in ALI has not yet been elucidated.

Methods: C57BL/6J mice were used in this study. ALI model was induced by intratracheal injection of lipopolysaccharide (LPS, $5 \mathrm{mg} / \mathrm{kg}$ ). The effects of Fn14 receptor blocker ATA ( $20 \mathrm{mg} / \mathrm{kg}$ ) on lung injury, inflammatory cell infiltration, inflammatory factor secretion, and oxidative stress in mice were observed. The activation of NLRP3 inflammasome was detected by qPCR, Western blot, and ELISA. Prophylactic or therapeutic ATA was administered to observe its effect on the survival rate of ALI mice. In vitro, primary mouse peritoneal macrophages were used to activate the NLRP3 inflammasome by LPS or LPS+ATP. Fn14 was activated by recombinant TWEAK, or knockdown by lentivirus, and the effects on NLRP3 inflammasome activation was detected.

Results: We found that ATA significantly downregulated the expression of Fn14 in the lungs and improved the survival rate of mice receiving a lethal dose of LPS. ATA also attenuated lung tissue damage by decreasing the infiltration of macrophages and neutrophils, reducing inflammation, and suppressing oxidative stress. Interestingly, we found that ATA strongly inhibited the activation of NLRP3 inflammasome in the lungs of ALI mice. Furthermore, in vitro, exogenous TWEAK, a natural ligand of Fn14, enhanced the levels of NLRP3 and Caspase-1 p10 and the maturation and secretion of IL-1 $\beta$ in the primary murine macrophages, eventually leading to the activation of NLRP3 inflammasome. In addition, the expression of Fn14, NLRP3, and Caspase-1 p10 and the production of IL-1 $\beta$ were effectively blocked by Fn14 shRNA in macrophages. In mechanism, the activation of Fn14 promoted the production of reactive oxygen species in activated macrophages.

Conclusion冈Our study first reports that the activation of Fn14 aggravates ALI by amplifying the activation of NLRP3 inflammasome. Therefore, blocking Fn14 may be a potential way to treat ALI.

\section{Background}

Acute lung injury (ALI) and its more severe form acute respiratory distress syndrome (ARDS) are the most common critical illnesses in clinical practice. ARDS is characterized by the development of uncontrolled pulmonary inflammation, disruptions of the alveolar-capillary barrier function, and non-cardiogenic pulmonary edema [1]. Particularly, the infiltration of inflammatory cells and increased pro-inflammatory cytokines and chemokines are the major pathological hallmarks of ALI. As the worldwide spread of SARS-CoV2, the research on the mechanism of ARDS is drawing more attention, as severe COVID-19 patients most end in ARDS [2]. Despite multiple diverse therapeutic methods, there is only a few effective therapies available to treat ALI. The mortality in critically ill patients remains $40 \%$ [3]. Better understanding the molecular basis of ALI may explore new anti-inflammatory drugs. 
The NLRs family, pyrin domain containing 3 (NLRP3) inflammasome, one of the pattern recognition receptors, plays a central role in the development of ALI [4]. The NLRP3 inflammasome is a multiprotein complex comprised of NLRP3, pro-caspase-1, and ASC. We have identified that inhibiting the activation of NLRP3 inflammasome alleviates LPS-induced ALI [5]. The activation of NLRP3 inflammasome includes two steps: priming and activation. Toll-like receptors (TLR) mediates the priming of NLRP3 inflammasome through the nuclear factor-KB (NF-KB) pathway. Damage-related molecular patterns, such as adenosine triphosphate (ATP), mediate the activation of NLRP3 inflammasome by inducing reactive oxygen species (ROS), ion flux (calcium ion influx or potassium ion outflow), or lysosomal detuning [6]. Moreover, activation of the NLRP3 inflammasome promotes the self-cleavage of Caspase- 1 precursor into activated Caspase-1 (Caspase-1 p10 and Caspase-1 p20), then mediates the maturation and secretion of IL-1 $\beta$ and IL-18 [7]. We have reported that vasoactive intestinal peptide and epoxyeicosatrienoic acids attenuate LPS-induced ALI in mice by targeting the activation of NLRP3 inflammasome $[5,8,9]$. However, the mechanism underlying the activation of NLRP3 inflammasome in LPS-induced ALI mice needs to be investigated.

Fibroblast growth factor-inducible 14 (Fn14) is the receptor of tumor necrosis factor-like weak inducer of apoptosis (TWEAK) and belongs to the TNF receptor superfamily [10]. Fn14 has a low level of ubiquitous expression on many cells. However, stress or inflammation could induce an increased expression. Fn14 is reportedly involved in acute and chronic inflammatory diseases, such as hepatitis, rheumatoid arthritis, and intestinal diseases [11-13]. In addition, the increased expression of Fn14 in the lungs of sepsis patients correlate with tissue damage [14]. However, whether Fn14 participates in ALI and its mechanism remains unclear. Fn14 activation could induce both the classical (canonical) pathway and the alternative (non-canonical) NF-KB signaling, leading to pro-inflammatory cytokines expression and contributing to tissue inflammation [15] Interestingly, Fn14 promotes the maturation and secretion of IL-1 [16]. Therefore, we assume that Fn14 participates in the cascade reaction of ALI, which may be related to NLRP3 inflammasome activation.

In this study, we investigated whether blocking Fn14 with an inhibitor (aurin tricarboxylic acid, ATA) [17] conferred protection against LPS-induced ALI. The study also could provide a new insight into the mechanism underlying the activation of NLRP3 inflammasome in macrophages during ALI.

\section{Materials And Methods}

\subsection{Animal}

Adult male C57BL/6J mice ( $20 \pm 2 \mathrm{~g}$ ) were purchased from Hunan SJA Laboratory Animal Co., Ltd. (Hunan, China). All animal experiments were approved by the Animal Care and Use Committee at Central South University and strictly followed guidelines regarding the care of laboratory animals.

\subsection{ALI model and animal treatment}


C57BL/6J mice were randomly divided into four groups: (a) the Control group, (b) ATA group, (c) ALI group, and (d) ALI+ATA group. Mice in the Control and ATA groups received $50 \mu \mathrm{L}$ sterile saline intratracheally. Mice in the ALI and ALI+ATA groups were given intrabronchial injection of LPS (E. coli 0111:B4; Sigma-Aldrich; USA) at $5 \mathrm{mg} / \mathrm{kg}$ dissolved in $50 \mu \mathrm{L}$ sterile saline. After intratracheal instillation, the mice were kept vertical for 5 min to ensure the distribution of the LPS or sterile in the lungs. In the ATA and ALI+ATA groups, ATA ( $20 \mathrm{mg} / \mathrm{kg}$ ) was intraperitoneally injected $1 \mathrm{~h}$ prior to the LPS instillation. All mice were sacrificed $12 \mathrm{~h}$ after LPS instillation and the lungs were collected and weighted. For survival study, the mice were intratracheally injected with LPS at a lethal dose $(25 \mathrm{mg} / \mathrm{kg})$. Saline or ATA (20 $\mathrm{mg} / \mathrm{kg}$ ) was intraperitoneally injected into mice $1 \mathrm{~h}$ before or after LPS administration. The survival rate was monitored every $6 \mathrm{~h}$. All surgical procedures were performed under $1.0 \%$ pentobarbital in sterile (80 $\mathrm{mg} / \mathrm{kg})$.

\subsection{Hematoxylin-eosin (HE) and inflammatory injury analysis}

The lung tissue samples were collected, fixed in 10\% formalin, embedded in paraffin, sectioned at $4 \mu \mathrm{m}$, and stained with HE. Histological scoring was performed blindly in line with four parts: alveolar congestion, hemorrhage, infiltration, and thickness of the alveolar wall/hyaline membrane formation. The lung injury score was measured as previously described [18]. The severity of the injury was graded from 0 to 4 , according to 5 variables: hyaline membranes, neutrophils in the alveolar space, hemorrhage, septal thickening, and pertinacious debris filling the airspaces.

\subsection{Lactate dehydrogenase (LDH) assay}

The activity of LDH in the serum of mice was determined by Lactate Dehydrogenase Activity Assay Kit (Jincheng Bioengineering Institute, Nanjing, China). Briefly, the serum samples were incubated with NADH and pyruvate for $15 \mathrm{~min}$ at $37^{\circ} \mathrm{C}$. Then, the enzymatic reaction was stopped by $0.4 \mathrm{~mol} / \mathrm{L} \mathrm{NaOH}$. Then, absorbance was measured at $450 \mathrm{~nm}$.

\subsection{Bronchoalveolar lavage fluid (BALF)}

BALF was collected with $0.8 \mathrm{~mL}$ of ice-cold PBS as previously described [19]. The BALF was centrifuged at $1500 \mathrm{rpm}$ for $5 \mathrm{~min}$. The pelleted cells were resuspended in PBS, and the cell numbers were counted. Protein concentration in BALF, an indicator of alveolar-capillary permeability, was measured using a BCA kit (Thermo Fisher Scientific). The number of total cells, macrophages, and neutrophils were counted with a hemocytometer and Wright-Giemsa staining.

\subsection{Measurement of myeloperoxidase (MPO)}

The lung tissue was weighed and homogenized. Then, according to the manufacturer's instructions, MPO activity in the lungs was measured by the MPO assay kit (Nanjing Jiancheng Bioengineering Institute, China). 


\subsection{Measurement of superoxide dismutase (SOD) and malondialdehyde (MDA)}

About 40 mg of left upper lung tissue was homogenated in cold PBS at a ratio of 1:10 (weight: volume). Protein concentration was determined using the BCA kit. The MDA level was measured by the thiobarbituric acid (TBA) method using a lipid peroxidation MDA assay kit (Jiancheng Biotech, Nanjing, Jiangsu, China) according to the manufacturer's instructions. Absorbance at $530 \mathrm{~nm}$ was measured by the microplate reader. SOD activity was evaluated by the xanthine oxidase method using a SOD activity assay kit (Jiancheng Biotech, Nanjing, Jiangsu, China) according to the manufacturer's instructions. Absorbance was measured at $450 \mathrm{~nm}$ by the microplate reader.

\subsection{Measurements of cytokine levels in the lungs}

The contents of tumor necrosis factor-alpha (TNF- $\alpha$ ), interlerkin-1 beta (IL-1 $\beta$ ), and monocyte chemoattractant protein-1 (MCP-1) in lung tissue, BALF, and cell culture supernatant were determined by specific enzyme-linked immunosorbent assay (ELISA) kits (Invitrogen; Thermo Fisher Scientific) according to the manufacturer's instructions.

\subsection{Real-time polymerase chain reaction (PCR)}

Total RNA was collected from lung tissue using RNAiso Plus (TaKaRa Clontech, Kusatsu, Japan) based on the manufacturer's instructions. RNA was quantified by measuring absorption at $260 \mathrm{~nm}$. The generation of cDNA from the total RNA $(1 \mu \mathrm{g})$ was performed with Prime Script RT reagent Kit with gDNA eraser (TaKaRa). Real-time PCR was performed with SYBR Fast qPCR mix (TaKaRa) on a Bio-Rad realtime PCR system (CFX96 Touch ${ }^{\text {TM }}$, Bio-Rad, USA). The expression levels (relative values) of the objective genes were calculated using the $2^{-\Delta \Delta C t}$ method [20]. $\beta$-actin was used as the internal control.

\subsection{Western blot analysis}

Total protein was extracted from mouse lungs and cells by RIPA. Protein concentration was detected by the bicinchoninic acid assay (BCA, Thermo Fisher Scientific). Equal amounts of protein $(30 \mu \mathrm{g})$ were loaded on SDS-PAGE and transferred to a polyvinylidene fluoride membrane [21]. After blocking with skim milk (5\%) in Tris-buffered saline (TBST) for $1 \mathrm{~h}$ at room temperature, the membrane was probed with the primary antibodies at $4^{\circ} \mathrm{C}$ overnight: rabbit anti-NLRP3 antibody (1:2000; CST, Danvers, MA), rabbit antiNrf2 antibody (1:1000, CST), goat anti-IL-1 $\beta$ antibody (1:2000; R\&D, Minneapolis, MN), rabbit anticaspase-1 antibody (1:1000; Abcam, Eugene, OR), rabbit anti-ASC antibody (1:1000, CST), rabbit antiFn14 antibody (1:1000, Abcam), rabbit anti- $\beta$-actin antibody (1:7500, Signal way Antibody, College Park, MD, USA), and rabbit anti-a-tubulin antibody (1:7500, Servicebio, China). After incubation with peroxidase-conjugated secondary antibodies (1:7500, Signal way Antibody) at room temperature for $1 \mathrm{~h}$, the signal was developed using an ECL chemiluminescence kit (Millipore, USA). The band intensities were quantitated using Image Lab software and normalized to internal reference values accordingly.

\subsection{Isolation and treatment of primary murine peritoneal macrophages}


The isolation and culture of primary murine peritoneal macrophages were conducted as described [22]. Four days after the intraperitoneal injection of $3 \mathrm{~mL} 3 \%$ thioglycolate (Sigma-Aldrich) into C57BL/6J mice, peritoneal macrophages were harvested by peritoneal lavage with precooled Roswell Park Memorial Institute RPMI 1640 (Gibco, Life Technologies, Carlsbad, CA). The cells were collected by centrifuging at $1000 \mathrm{rpm}$ for $8 \mathrm{~min}$ and washed with cooled phosphate-buffered saline. The cells were resuspended in cell culture medium and counted on the counting plate after sufficient mixing. Macrophages were plated into 6-well or 12 -well plates $\left(2 \times 10^{6}\right.$ cells/well or $1 \times 10^{6}$ cells/well). To investigate the effect of rTWEAK on the macrophages, the cells were treated with rTWEAK $(100 \mathrm{ng} / \mathrm{mL})$ and LPS $(100 \mathrm{ng} / \mathrm{mL})$ for $12 \mathrm{~h}$. To investigate the activation of NLRP3 inflammasome, the cells were primed with rTWEAK $(100 \mathrm{ng} / \mathrm{mL})$ and LPS $(100 \mathrm{ng} / \mathrm{mL})$ for $135 \mathrm{~min}$. Cells were then stimulated with ATP $(2.5 \mathrm{mM}$, Solarbio, China) for $45 \mathrm{~min}$.

\subsection{Measurement of ROS}

The primary peritoneal macrophages $\left(2 \times 10^{6}\right.$ cells/well) were seeded into the 6-well plates. Dilute DCFHDA (Nanjing Jiancheng Bioengineering Institute, China) with RPMI 1640 at the ratio of 1:1000 to a final concentration of $10 \mu \mathrm{M}$. Add $1 \mathrm{~mL}$ diluted DCFH-DA solution to each well and incubate for $20 \mathrm{~min}$ at 37 ${ }^{\circ} \mathrm{C}$. The cell suspension was obtained by adding $1 \mathrm{~mL}$ RPMI 1640, then was centrifuged at $1500 \mathrm{rpm}$ for 5 min, followed by resuspension with $400 \mu \mathrm{L}$ PBS for flow cytometry assay (BD, USA).

\subsection{Silenced the expression of Fn14 by shRNA}

The short hairpin RNA (shRNA) of Fn14 was purchased from Gene Chen (Shanghai, China). Fn14 shRNA sequences were gattcggcttggtgttgatgc. Macrophages were infected with concentrated lentivirus. The supernatant was replaced with complete culture medium after $16 \mathrm{~h}$. After treated with shRNA, the cells were primed with LPS $(100 \mathrm{ng} / \mathrm{mL})$ for $135 \mathrm{~min}$. Cells were then stimulated with ATP $(2.5 \mathrm{mM}$, Solarbio, China) for $45 \mathrm{~min}$.

\subsection{Statistical analysis}

All experiments were independently repeated three times. Data were presented as means \pm SEM and conducted using SPSS 21. Differences between the two groups were analyzed with a $t$-test. Differences between multiple groups were analyzed using ANOVA, followed by Tukey's post hoc test. The survival rate was assayed by the log-rank test. A value of $P<0.05$ was considered significant.

\section{Results}

\subsection{Fn14 expression was increased in the lung of ALI mice}

We first investigated whether Fn14 expression was correlated with ALI. The mRNA and protein expression of Fn14 were raised in the lungs of LPS-induced ALI mice (Figure 1A-C). These data suggest that Fn14 may be a key orchestrator in the pathogenesis of ALI. 


\subsection{Blockade of Fn14 by ATA alleviates LPS-induced lung injury in mice}

ATA was previously identified as a novel inhibitor of Fn14 signaling [17]. Here, we adopted ATA to investigate the function of Fn14 function in ALI. The protein expression of Fn14 in the lungs of ALI mice was downregulated after pre-treatment with ATA $(20 \mathrm{mg} / \mathrm{kg}) 1 \mathrm{~h}$ before LPS administration (Figure 2A-B). Administration of ATA significantly prevented the death of mice receiving $25 \mathrm{mg} / \mathrm{kg}$ LPS (Figure 2C). H\&E staining indicated the accumulation of inflammatory cells, destruction of the alveolar histological structure, and the collapse of the alveoli in LPS-treated mice. Remarkably, ATA pre-treatment alleviated those pathological changes (Figure 2D-E). LDH release is positively related to cellular damage. We found that ATA pre-treatment pronounced reduced the LDH activity in the serum (Figure 2F). In parallel, the W/D ratio of lung tissue was significantly decreased in ATA-treated mice (Figure 2G). ATA pre-treatment robustly reduced the number of total cells, macrophages, and neutrophils in the BALF (Figure $2 \mathrm{H}-\mathrm{J}$ ) and MPO activity in the lung (Figure $2 \mathrm{~K}$ ), which reflects neutrophil accumulation in the lungs. Taken together, these data indicate that blockade of Fn14 mitigates lung damage in ALI mice.

\subsection{Blockade of Fn14 by ATA inhibits the inflammatory responses and oxidative stress in ALI mice}

Inflammation and oxidative stress are dispensable characteristics of ALI. We found that ATA pretreatment reduced the pro-inflammatory cytokines and chemokines mRNA expression, including Tnf- $a$ and Trem-1 (Figure 3A, D). Protein expression of TNF-a in BALF and lungs was decreased in ATA-treated mice (Figure 3B-C). MCP-1 recruits monocytes to the site of inflammation. MCP-1 mRNA and protein expression were also down-regulated by ATA treatment (Figure 3E-F). In addition, ATA pre-treatment reduced the MDA formation in the lungs of ALI mice (Figure 3G). In contrast, ATA pre-treatment remarkably restored the activity of the antioxidative enzyme SOD in the lungs (Figure $3 \mathrm{H}$ ). Nuclear factorerythroid 2-related factor 2 (Nrf2) is a key transcription factor in the antioxidative stress response of cells. ATA pre-treatment remarkably increased the Nrf2 protein expression (Figure 3l-J). Collectively, these results suggest that blockade of Fn14 inhibits inflammatory responses and oxidative stress in LPSinduced ALI mice.

\subsection{Blockade of Fn14 by ATA improves the survival of LPS- treated mice}

To explore the therapeutic potential of ATA, we investigated the potential benefits of ATA treatment (1 $\mathrm{g} / \mathrm{kg}) 2 \mathrm{~h}$ post-LPS administration. We found that treatment with ATA $2 \mathrm{~h}$ post-LPS exposure also significantly improved the survival rate of LPS-treated mice (Figure 4). This result indicates that blockade of Fn14 shows therapeutic benefits to LPS-induced ALI in mice. 


\subsection{Blockade of Fn14 by ATA inhibits the NLRP3 inflammasome activation in ALI mice}

The NLRP3 inflammasome plays a vital role in the pathogenesis of ALI [5]. We first investigated whether blockade of Fn14 with ATA repressed the activation of NLRP3 inflammasome in vivo. Results showed that ATA profoundly reduced the expression of Asc, NIrp3 and pro-Il-1 $\beta$ mRNA in the lungs of ALI mice (Figure 5A-C). Upon NLRP3 inflammasome activation, pro-caspase-1 is hydrolyzed into Caspase-1 p10 and Caspase-1p20, cleaving pro-IL-1 $\beta$ into IL-1 $\beta$. We found ATA remarkably decreased the NLRP3, Caspase-1 p20, and Caspase-1 p10 protein levels in the lungs of ALI mice (Figure 5D-G). NLRP3 inflammasome activation gives rise to the secretion of active IL-1 $\beta$. The results showed that ATA pretreatment effectively reduced the IL-1 $\beta$ in BALF and lungs of ALI mice (Figure $5 \mathrm{H}-\mathrm{I}$ ). These data suggest that the ATA inhibits the activation of NLRP3 inflammasome in the lungs of ALI mice.

\subsection{Activation of Fn14 by rTWEAK promotes the priming of NLRP3 inflammasome in macrophages}

Next, we investigated the effects of Fn14 on the NLRP3 inflammasome priming in primary macrophages. rTWEAK, a natural ligand of FN14, was used to stimulate primary peritoneal macrophages. Interestingly, we found that, compared with the LPS group, rTWEAK significantly increased the secretion of IL-1 $1 \beta$, but not TNF-a (Figure 6A-B), indicating that rTWEAK specifically amplifies NLRP3 inflammasome-mediated inflammatory response. Further, we found that LPS-induced upregulation of NLRP3, pro-caspase-1, pro-IL$1 \beta$, and ASC expression. The rTWEAK treatment significantly increased the expression of NLRP3, procaspase-1, pro-IL-1 $\beta$, and ASC (Figure $6 \mathrm{C}-\mathrm{G}$ ). These results suggest that activation of Fn14 promotes the priming of NLRP3 inflammasome.

\section{7 rTWEAK pre-treatment aggravates the activation of NLRP3 inflammasome in macrophages}

We treated macrophages with LPS plus ATP to activate the NLRP3 inflammasome. We found that pretreatment of rTWEAK significantly increased the levels of Caspase-1 p10 and Caspase-1 p20 in macrophages treated by LPS with ATP (Figure 7A-C). IL-1 13 p17 level in the cells and supernatant was also increased upon rTWEAK treatment (Figure 7D-G). In addition, we silenced the expression of Fn14 in macrophages. Fn14 shRNA significantly decreased the Fn14 and NLRP3 expression induced by LPS and ATP (Figure 8A-C). Furthermore, the expression of Caspase-1 p10 was effectively blocked by Fn 14 shRNA in macrophages (Figure 8D-E). IL-1 $\beta$ production was effectively blocked by Fn14 shRNA in macrophages (Figure 8F). These data collectively suggest that activation of Fn14 by rTWEAK aggravates the activation of NLRP3 inflammasome in macrophages.

\section{8 rTWEAK pre-treatment promotes the ROS production in macrophages}


Given that excessive ROS mediates NLRP3 inflammasome activation, we investigated whether activation of Fn14 could increase ROS production. As demonstrated in Figure 9, after treatment with LPS + ATP, an increase in the DCF fluorescence intensity was observed in primary macrophages. Pre-treatment of rTWEAK significantly increased the production of ROS in the primary macrophages (Figure 9). The results confirm that rTWEAK promotes the ROS production, contributing to the activation of NLRP3 inflammasome in primary macrophages.

\section{Discussion}

In this study, we found blocking Fn14 reduced LPS-induced ALI in mice. Blockade of Fn14 suppressed the activation of NLRP3 inflammasome in vivo. In vitro, Fn14 activation amplified the activation of NLRP3 inflammasome in primary murine macrophages. Our study implies that Fn14 may play a key role in the pathogenesis of ALI and provides a novel mechanism of Fn14 activation on the inflammatory cascade.

This study identifies that elevated Fn14 is a novel mechanism in the uncontrolled inflammatory cascade during ALI. Fn14 is a type I transmembrane protein encoded by fibroblast growth factors-regulating early response gene [23]. Studies have reported that $\mathrm{Fn} 14$ is significantly up-regulated in inflammation-related diseases, such as intestinal diseases, kidney disease, accelerating the course of illness [24,25]. Our study found that Fn14 expression was significantly increased in LPS-induced ALI in mice. ATA, an inhibitor of Fn14, blocks the TWEAK/Fn14 signal axis by inhibiting the binding of TRAF2 [17], reduced lung tissue pathology injury, and decreased the number of inflammatory cells and pro-inflammatory cytokines factors such as TNF-a and MCP-1 in BALF. More importantly, blockade of Fn14 activation effectively improved the survival rate of mice treated by a lethal dose of LPS. These results suggest that blocking Fn14 activation alleviates the LPS-induced ALI. In another study, blockade of Fn14 on pulmonary microvascular endothelial cells (PMVECs) shows a protective effect against cecal ligation and punctureinduced ALI [14]. We speculated, therefore, that Fn14 may be an innovative target for the treatment of ALI.

We reveal a new function of Fn14 in macrophages. Fn14 could express in different types of cells, such as macrophages, fibroblast [26], PMVECs [14], vascular smooth muscle cells [27], and cancer cells [28]. Previous studies have reported that Fn14 expressed on macrophages promotes oxidative stress through NADPH oxidase activation [29] or increases HMGB1 expression and secretion [30]. Herein, we found Fn14 amplifies the activation of NLRP3 inflammasome in macrophages. Blocking Fn14 activation inhibited the expression of NLRP3 inflammasome components, including NLRP3, ASC, pro-caspase-1, pro-IL-1 $\beta$, and secretion of IL-1 $\beta$ in LPS-induced ALI mice. The accumulation of evidence points to the central role of NLRP3 inflammasome in the ALI [4, 31]. Interestingly, activation of Fn14 with rTWEAK fails to increase the TNF-a secretion, indicating that Fn14 specifically amplifies NLRP3 inflammasome-mediated inflammatory response. Therefore, we propose for the first time that activated Fn14 aggravates pulmonary inflammation by activating NLRP3 inflammasome, which may be a new mechanism explaining the uncontrolled inflammation in ALI. 
Oxidative stress is the crucial pathogenesis of ALI. TWEAK-Fn14 axis is directly involved in oxidative stress. Our results indicate that ATA alleviates oxidative stress in the lungs of ALI mice and enhances the antioxidant capacity by up-regulating the expression of Nrf2 and SOD. It has been reported that excessive production of ROS induced by TWEAK could be eliminated by genetic silencing of Fn14 in macrophages [29]. Furthermore, TWEAK/Fn14 could promote oxidative stress in the AMPK/PGC1-a-dependent manner [32]. However, the underlying mechanism remains obscure.

Interestingly, there was no significant increase of TWEAK expression in the lungs of ALI mice, indicating that Fn14 may execute its function independent of its ligand TWEAK. Studies have also confirmed that the pro-inflammatory effect of TWEAK is mainly dependent on Fn14. The pro-inflammatory effect of TWEAK disappears after treatment with Fn14-siRNA [33]. While, Fn14 may interact with other mediators, mediating tissue inflammation. It has been reported that TWEAK was found to have no significant change in $\mathrm{CCl}_{4}$-induced acute liver injury [34]. Even though, we observed that $\mathrm{Fn} 14$ expression was significantly up-regulated in ALI, indicating a pro-inflammatory role in the ALI process.

In summary, our study indicates that the increased expression of Fn14 expression is a novel pathological mechanism in ALI. Blocking Fn14 effectively ameliorates LPS-induced ALI in mice. TWEAK/Fn14 axis activates the NLRP3 inflammasome, contributing to the uncontrolled inflammation response in ALI.

\section{Declarations}

\section{Funding}

This work was supported by the National Natural Science Foundation of China (91949110, 82170096), the Open Project of the State Key Laboratory of Trauma, Burns, and Combined Injury, Army Medical University (SKLKF201702), High School Innovation Fund of Hunan province (19K103), and Fundamental Research Funds for the Central Universities of Central South University (2019zzts318).

\section{Conflicts of interest}

The authors confirm that the content of this article has no conflicts of interest.

\section{Author contributions}

X.X.G., H.H.Y., W.J.Z., C.Y.Z., and H.L.J. performed the experiments; X.X.G., J.X.D., and H.H.Y. analyzed the data; Y.Z., C.X.G., and Y.X contributed reagents/materials/analysis tools; X.X.G. and W.J.Z. wrote the paper; C.X.G. and Y.Z. conceived, designed the experiments, and critically reviewed the manuscript. All authors had final approval of the submitted versions.

\section{Consent for publication}

Not applicable. 


\section{Ethics approval and consent to participate}

All animal experiments were approved by the Ethics Committee of the School of Basic Medical Science, Central South University (2020sydw0685, Changsha, China).

\section{Availability of data and materials}

The datasets generated during and/or analyzed during the current study are available from the corresponding author on reasonable request.

\section{Acknowledgements}

The authors would like to thank all the institutions and researchers who contributed to this study.

\section{References}

1. Fan, E., Brodie, D. \& Slutsky, A. S. (2018) Acute Respiratory Distress Syndrome: Advances in Diagnosis and Treatment, JAMA. 319, 698-710.

2. Xu, Z., Shi, L., Wang, Y., Zhang, J., Huang, L., Zhang, C., Liu, S., Zhao, P., Liu, H., Zhu, L., Tai, Y., Bai, C., Gao, T., Song, J., Xia, P., Dong, J., Zhao, J. \& Wang, F. S. (2020) Pathological findings of COVID-19 associated with acute respiratory distress syndrome, Lancet Respir Med. 8, 420-422.

3. Matthay, M. A., Zemans, R. L., Zimmerman, G. A., Arabi, Y. M., Beitler, J. R., Mercat, A., Herridge, M., Randolph, A. G. \& Calfee, C. S. (2019) Acute respiratory distress syndrome, Nat Rev Dis Primers. 5, 18.

4. Zhang, Y., Li, X., Grailer, J. J., Wang, N., Wang, M., Yao, J., Zhong, R., Gao, G. F., Ward, P. A., Tan, D. X. \& $\mathrm{Li}, \mathrm{X}$. (2016) Melatonin alleviates acute lung injury through inhibiting the NLRP3 inflammasome, $J$ Pineal Res. 60, 405-14.

5. Yang, H. H., Duan, J. X., Liu, S. K., Xiong, J. B., Guan, X. X., Zhong, W. J., Sun, C. C., Zhang, C. Y., Luo, X. Q., Zhang, Y. F., Chen, P., Hammock, B. D., Hwang, S. H., Jiang, J. X., Zhou, Y. \& Guan, C. X. (2020) A COX-2/sEH dual inhibitor PTUPB alleviates lipopolysaccharide-induced acute lung injury in mice by inhibiting NLRP3 inflammasome activation, Theranostics. 10, 4749-4761.

6. Swanson, K. V., Deng, M. \& Ting, J. P. (2019) The NLRP3 inflammasome: molecular activation and regulation to therapeutics, Nat Rev Immunol. 19, 477-489.

7. Pinar, A. A., Scott, T. E., Huuskes, B. M., Tapia Caceres, F. E., Kemp-Harper, B. K. \& Samuel, C. S. (2020) Targeting the NLRP3 inflammasome to treat cardiovascular fibrosis, Pharmacol Ther. 209, 107511.

8. Zhou, Y., Zhang, C. Y., Duan, J. X., Li, Q., Yang, H. H., Sun, C. C., Zhang, J., Luo, X. Q. \& Liu, S. K. (2020) Vasoactive intestinal peptide suppresses the NLRP3 inflammasome activation in lipopolysaccharideinduced acute lung injury mice and macrophages, Biomed Pharmacother. 121, 109596.

9. Luo, X. Q., Duan, J. X., Yang, H. H., Zhang, C. Y., Sun, C. C., Guan, X. X., Xiong, J. B., Zu, C., Tao, J. H., Zhou, Y. \& Guan, C. X. (2020) Epoxyeicosatrienoic acids inhibit the activation of NLRP3 inflammasome in murine macrophages, J Cell Physiol. 235, 9910-9921. 
10. Winkles, J. A. (2008) The TWEAK-Fn14 cytokine-receptor axis: discovery, biology and therapeutic targeting, Nat Rev Drug Discov. 7, 411-25.

11. Affo, S., Dominguez, M., Lozano, J. J., Sancho-Bru, P., Rodrigo-Torres, D., Morales-lbanez, O., Moreno, M., Millan, C., Loaeza-del-Castillo, A., Altamirano, J., Garcia-Pagan, J. C., Arroyo, V., Gines, P., Caballeria, J., Schwabe, R. F. \& Bataller, R. (2013) Transcriptome analysis identifies TNF superfamily receptors as potential therapeutic targets in alcoholic hepatitis, Gut. 62, 452-60.

12. van Kuijk, A. W., Wijbrandts, C. A., Vinkenoog, M., Zheng, T. S., Reedquist, K. A. \& Tak, P. P. (2010) TWEAK and its receptor Fn14 in the synovium of patients with rheumatoid arthritis compared to psoriatic arthritis and its response to tumour necrosis factor blockade, Ann Rheum Dis. 69, 301-4.

13. Di Martino, L., Dave, M., Menghini, P., Xin, W., Arseneau, K. O., Pizarro, T. T. \& Cominelli, F. (2016) Protective Role for TWEAK/Fn14 in Regulating Acute Intestinal Inflammation and Colitis-Associated Tumorigenesis, Cancer Res. 76, 6533-6542.

14. Zou, Y., Bao, S., Wang, F., Guo, L., Zhu, J., Wang, J., Deng, X. \& Li, J. (2018) FN14 Blockade on Pulmonary Microvascular Endothelial Cells Improves the Outcome of Sepsis-Induced Acute Lung Injury, Shock. 49, 213-220.

15. Sanz, A. B., Sanchez-Nino, M. D. \& Ortiz, A. (2011) TWEAK, a multifunctional cytokine in kidney injury, Kidney Int. 80, 708-18.

16. Xu, J., He, J., He, H., Peng, R. \& Xi, J. (2017) TWEAK-Fn14 Influences Neurogenesis Status via Modulating NF-kappaB in Mice with Spinal Cord Injury, Mol Neurobiol. 54, 7497-7506.

17. Zhang, J., Dong, B., Hao, J., Yi, S., Cai, W. \& Luo, Z. (2019) LncRNA Snhg3 contributes to dysfunction of cerebral microvascular cells in intracerebral hemorrhage rats by activating the TWEAK/Fn14/STAT3 pathway, Life Sci. 237, 116929.

18. Duan, J. X., Zhou, Y., Zhou, A. Y., Guan, X. X., Liu, T., Yang, H. H., Xie, H. \& Chen, P. (2017) Calcitonin gene-related peptide exerts anti-inflammatory property through regulating murine macrophages polarization in vitro, Mol Immunol. 91, 105-113.

19. Zhou, Y., Liu, T., Duan, J. X., Li, P., Sun, G. Y., Liu, Y. P., Zhang, J., Dong, L., Lee, K. S. S., Hammock, B. D., Jiang, J. X. \& Guan, C. X. (2017) Soluble Epoxide Hydrolase Inhibitor Attenuates LipopolysaccharideInduced Acute Lung Injury and Improves Survival in Mice, Shock. 47, 638-645.

20. Zhang, C. Y., Duan, J. X., Yang, H. H., Sun, C. C., Zhong, W. J., Tao, J. H., Guan, X. X., Jiang, H. L., Hammock, B. D., Hwang, S. H., Zhou, Y. \& Guan, C. X. (2020) COX-2/sEH dual inhibitor PTUPB alleviates bleomycin-induced pulmonary fibrosis in mice via inhibiting senescence, FEBS J. 287, 1666-1680.

21. Zhou, Y., Yang, J., Sun, G. Y., Liu, T., Duan, J. X., Zhou, H. F., Lee, K. S., Hammock, B. D., Fang, X., Jiang, J. X. \& Guan, C. X. (2016) Soluble epoxide hydrolase inhibitor 1-trifluoromethoxyphenyl-3- (1propionylpiperidin-4-yl) urea attenuates bleomycin-induced pulmonary fibrosis in mice, Cell Tissue Res. 363, 399-409.

22. Zhong, W. J., Yang, H. H., Guan, X. X., Xiong, J. B., Sun, C. C., Zhang, C. Y., Luo, X. Q., Zhang, Y. F., Zhang, J., Duan, J. X., Zhou, Y. \& Guan, C. X. (2019) Inhibition of glycolysis alleviates 
lipopolysaccharide-induced acute lung injury in a mouse model, J Cell Physiol. 234, 4641-4654.

23. Mendez-Barbero, N., Yuste-Montalvo, A., Nunez-Borque, E., Jensen, B. M., Gutierrez-Munoz, C., TomeAmat, J., Garrido-Arandia, M., Diaz-Perales, A., Ballesteros-Martinez, C., Laguna, J. J., Beitia, J. M., Poulsen, L. K., Cuesta-Herranz, J., Blanco-Colio, L. M. \& Esteban, V. (2020) The TNF-like weak inducer of the apoptosis/fibroblast growth factor-inducible molecule 14 axis mediates histamine and platelet-activating factor-induced subcutaneous vascular leakage and anaphylactic shock, J Allergy Clin Immunol. 145, 583-596 e6.

24. Dohi, T., Borodovsky, A., Wu, P., Shearstone, J. R., Kawashima, R., Runkel, L., Rajman, L., Dong, X., Scott, M. L., Michaelson, J. S., Jakubowski, A. \& Burkly, L. C. (2009) TWEAK/Fn14 pathway: a nonredundant role in intestinal damage in mice through a TWEAK/intestinal epithelial cell axis, Gastroenterology. 136, 912-23.

25. Poveda, J., Vazquez-Sanchez, S., Sanz, A. B., Ortiz, A., Ruilope, L. M. \& Ruiz-Hurtado, G. (2021) TWEAK-Fn14 as a common pathway in the heart and the kidneys in cardiorenal syndrome, $J$ Pathol.

26. Zhang, Y., Zeng, W. \& Xia, Y. (2021) TWEAK/Fn14 axis is an important player in fibrosis, J Cell Physiol. 236, 3304-3316.

27. Munoz-Garcia, B., Madrigal-Matute, J., Moreno, J. A., Martin-Ventura, J. L., Lopez-Franco, O., Sastre, C., Ortega, L., Burkly, L. C., Egido, J. \& Blanco-Colio, L. M. (2011) TWEAK-Fn14 interaction enhances plasminogen activator inhibitor 1 and tissue factor expression in atherosclerotic plaques and in cultured vascular smooth muscle cells, Cardiovasc Res. 89, 225-33.

28. Dwyer, B. J., Jarman, E. J., Gogoi-Tiwari, J., Ferreira-Gonzalez, S., Boulter, L., Guest, R. V., Kendall, T. J., Kurian, D., Kilpatrick, A. M., Robson, A. J., O'Duibhir, E., Man, T. Y., Campana, L., Starkey Lewis, P. J., Wigmore, S. J., Olynyk, J. K., Ramm, G. A., Tirnitz-Parker, J. E. E. \& Forbes, S. J. (2021) TWEAK/Fn14 signalling promotes cholangiocarcinoma niche formation and progression, J Hepatol. 74, 860-872.

29. Madrigal-Matute, J., Fernandez-Laso, V., Sastre, C., Llamas-Granda, P., Egido, J., Martin-Ventura, J. L., Zalba, G. \& Blanco-Colio, L. M. (2015) TWEAK/Fn14 interaction promotes oxidative stress through NADPH oxidase activation in macrophages, Cardiovasc Res. 108, 139-47.

30. Moreno, J. A., Sastre, C., Madrigal-Matute, J., Munoz-Garcia, B., Ortega, L., Burkly, L. C., Egido, J., Martin-Ventura, J. L. \& Blanco-Colio, L. M. (2013) HMGB1 expression and secretion are increased via TWEAK-Fn14 interaction in atherosclerotic plaques and cultured monocytes, Arterioscler Thromb Vasc Biol. 33, 612-20.

31. Liu, T., Zhou, Y., Li, P., Duan, J. X., Liu, Y. P., Sun, G. Y., Wan, L., Dong, L., Fang, X., Jiang, J. X. \& Guan, C. X. (2016) Blocking triggering receptor expressed on myeloid cells-1 attenuates lipopolysaccharideinduced acute lung injury via inhibiting NLRP3 inflammasome activation, Sci Rep. 6, 39473.

32. Liu, H., Peng, H., Xiang, H., Guo, L., Chen, R., Zhao, S., Chen, W., Chen, P., Lu, H. \& Chen, S. (2018) TWEAK/Fn14 promotes oxidative stress through AMPK/PGC1alpha/MnSOD signaling pathway in endothelial cells, Mol Med Rep. 17, 1998-2004.

33. Sidler, D., Wu, P., Herro, R., Claus, M., Wolf, D., Kawakami, Y., Kawakami, T., Burkly, L. \& Croft, M. (2017) TWEAK mediates inflammation in experimental atopic dermatitis and psoriasis, Nat Commun. $\mathbf{8}$, 
15395.

34. Wilhelm, A., Shepherd, E. L., Amatucci, A., Munir, M., Reynolds, G., Humphreys, E., Resheq, Y., Adams, D. H., Hubscher, S., Burkly, L. C., Weston, C. J. \& Afford, S. C. (2016) Interaction of TWEAK with Fn14 leads to the progression of fibrotic liver disease by directly modulating hepatic stellate cell proliferation, J Pathol. 239, 109-21.

\section{Tables}

Table 1. Sequences of specific primers (mouse) used in this study.

\begin{tabular}{|lll|}
\hline Gene & Forward primer (5' to $\left.3^{\prime}\right)$ & Reverse primer (5' to $\left.3^{\prime}\right)$ \\
\hline Mcp-1 & GTCCCTGTCATGCTTCTGG & GCGTTAACTGCATCTGGCT \\
\hline Tnf- $a$ & CCACCCGCTCTTCTGTCTA & TGGTTTGTGAGTGAGGGT \\
\hline Pro-II-1 $\beta$ & CAGGCAGGCAGTATCACTCA & AGCTCATATGGGTCCGACAG \\
\hline Trem-1 & CTGTGCGTGTTCTTTGTC & CTTCCCGTCTGGTAGTCT \\
\hline Asc & GACAGTACCAGGCAGTTCGT & AGTCCTTGCAGGTCAGGTTC \\
\hline NIrp3 & TACGGCCGTCTACGTCTTCT & CGCAGATCACACTCCTCAAA \\
\hline Fn14 & CAGATCCTCGTGTTGGGATT & AACTAGAAACCAGCGCCAAA \\
\hline$\beta$-actin & CACCATGTACCCAGGCATTG & CCTGCTTGCTGATCCACATC \\
\hline
\end{tabular}

\section{Figures}
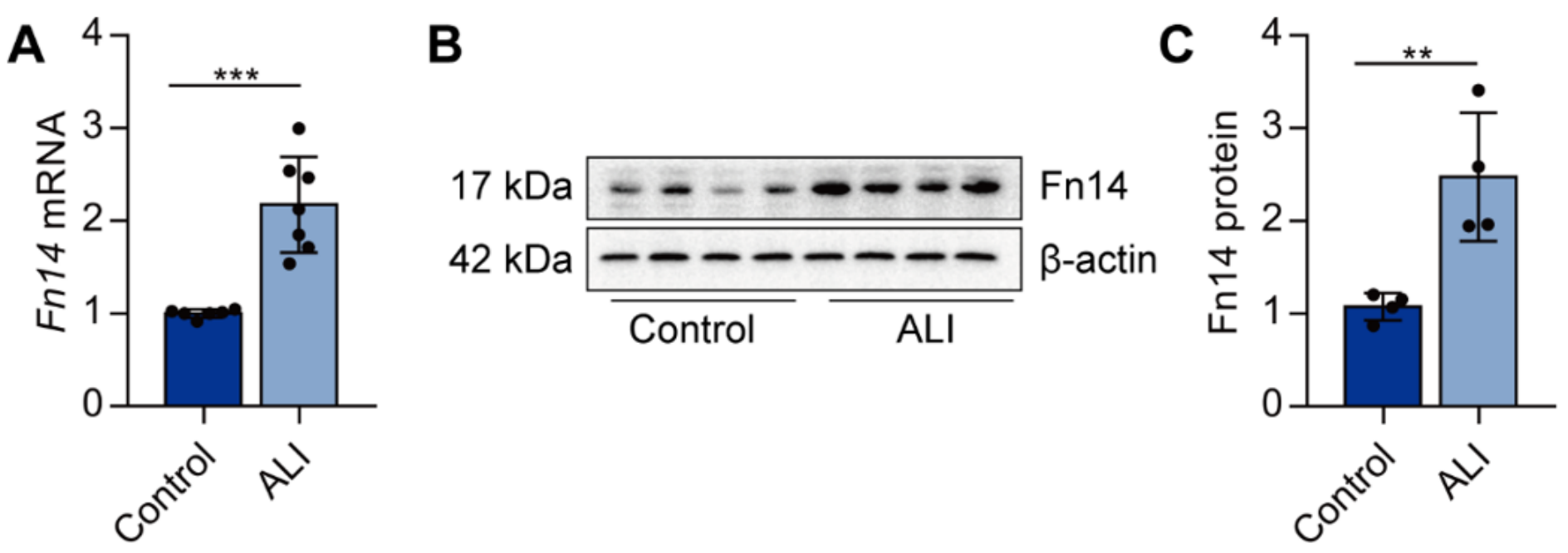

Figure 1 
Fn14 expression is increased in the lung of ALI mice. C57BL/6J mice were intraperitoneally injected with LPS (5 mg/kg, i.t.). Twelve hours later, the expression of Fn14 mRNA in the lung was determined by realtime PCR $(A, n=7)$. The protein expression of Fn14 in the lungs was determined by Western blot $(B-C$, $\mathrm{n}=4) .{ }^{* * \mathrm{P}}<0.01$ and ${ }^{* * *} \mathrm{P}<0.001$.

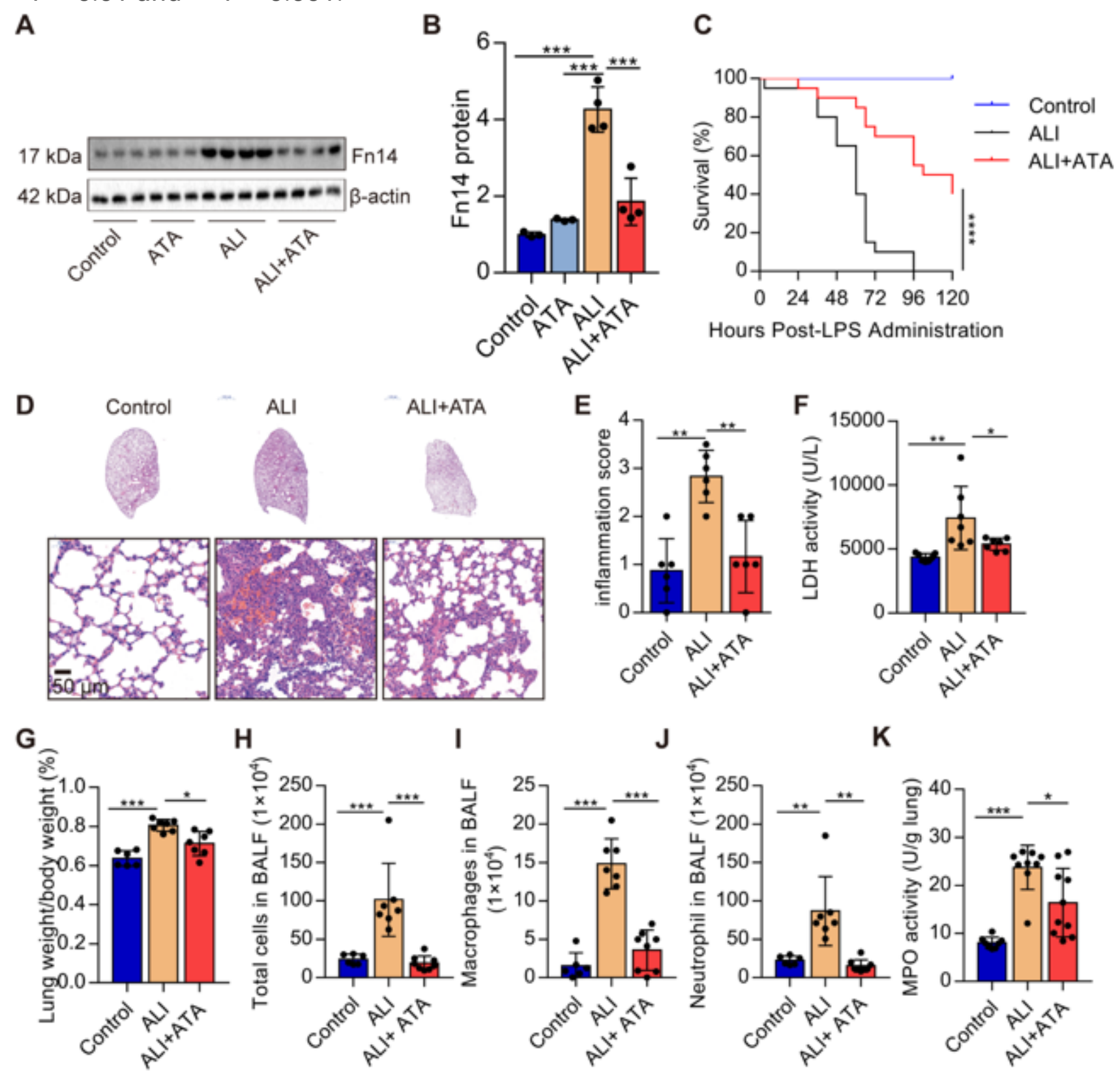

Figure 2

Blockade of Fn14 by ATA alleviates LPS-induced ALI in mice. ATA $(20 \mathrm{mg} / \mathrm{kg})$ was administered to mice 1 $\mathrm{h}$ before induction of ALI by LPS (5 mg/kg). (A-B) The protein expression of Fn14 in the lungs was determined by Western blot $(n=4)$. (C) The mortality was monitored every 6 h over $120 \mathrm{~h}(n=20$ per group). C57BL $/ 6 \mathrm{~J}$ mice were treated with saline or ATA $(20 \mathrm{mg} / \mathrm{kg})$. One hour later, mice were given saline or LPS ( $5 \mathrm{mg} / \mathrm{kg}$, i.t.). (D) Twelve hours later, the lung histopathological change was determined by H\&E staining $(B a r=50 \mu \mathrm{m})$. (E) The change of lung inflammation score was determined $(n=6)$. (F) The activity of LDH in BALF was determined $(n=6-7)$. $(G)$ The ratio of lung weight/body weight was determined $(n=$ 
6-7). (H-J) The Quantification of total cells, macrophages, and neutrophils in BALF was determined $(n=6-$ 7). (K) The activity of MPO in lung tissue was determined $(n=6-10) .{ }^{*} P<0.05,{ }^{*} P<0.01$, and ${ }^{\star \star *} P<$ 0.001 .
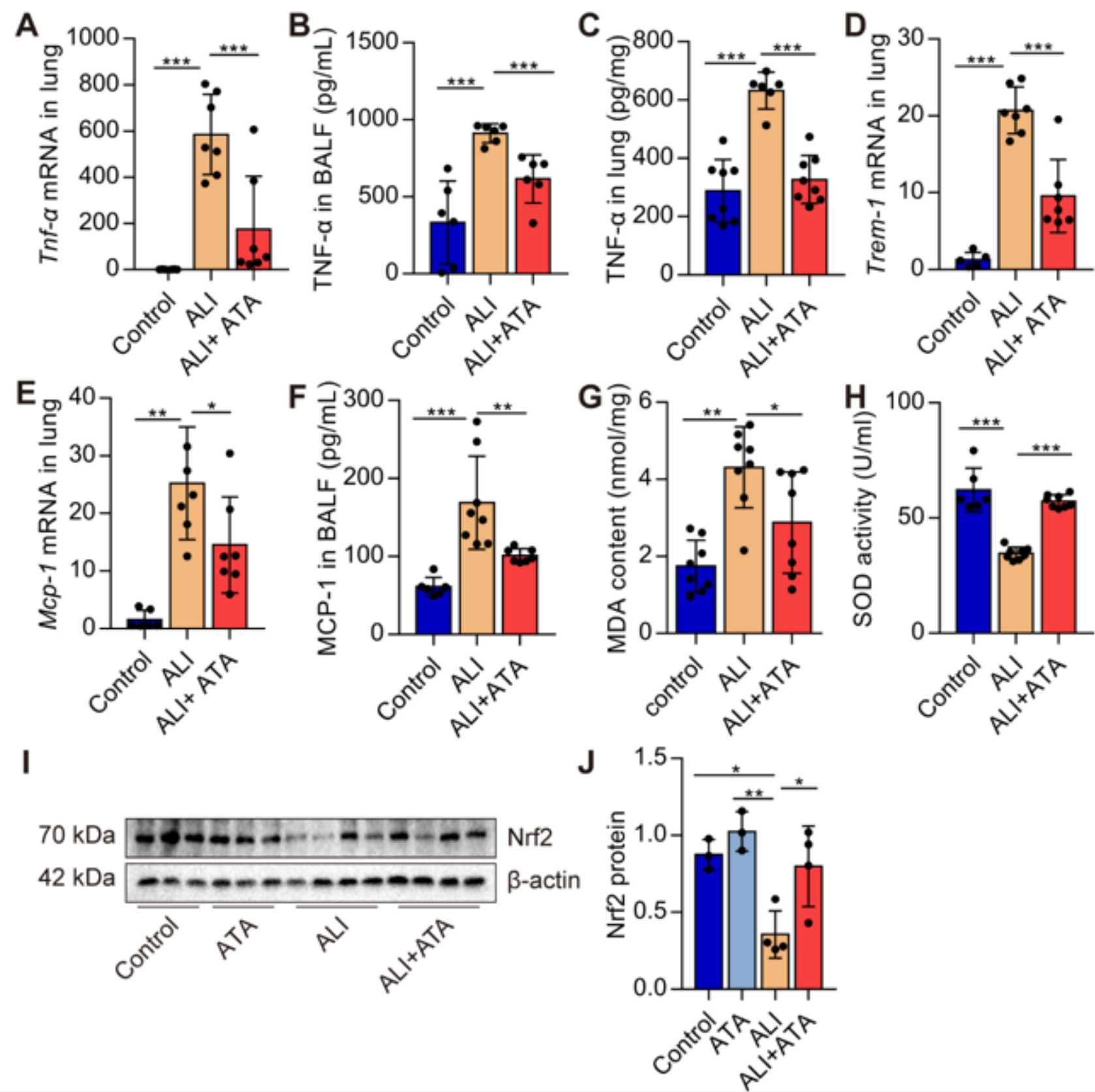

\section{Figure 3}

Blockade of Fn14 by ATA reduces the inflammatory responses and oxidative stress in ALI mice.

C57BL/6J mice were treated with saline or ATA $(20 \mathrm{mg} / \mathrm{kg})$. One hour later, mice were given saline or LPS (5 mg/kg, i.t.). (A-C) Twelve hours later, TNF-a mRNA and protein levels were determined by real-time PCR and ELISA $(n=6-9)$. (D) The expression of Trem-1 mRNA was determined by real-time PCR $(n=5-7)$. (E-F) The MCP-1 mRNA and protein were determined by real-time PCR and ELISA $(n=6-8)$. (G) The content of MDA in the lungs was determined $(n=8)$. $(H)$ The activity of SOD in lung tissue was determined $(n=6-8)$. 
$(I-J)$ The protein expression of Nrf2 in the lungs was determined by Western blot $(n=3-4) .{ }^{*}<0.05,{ }^{* \star} P<$ 0.01 , and $* * * P<0.001$.

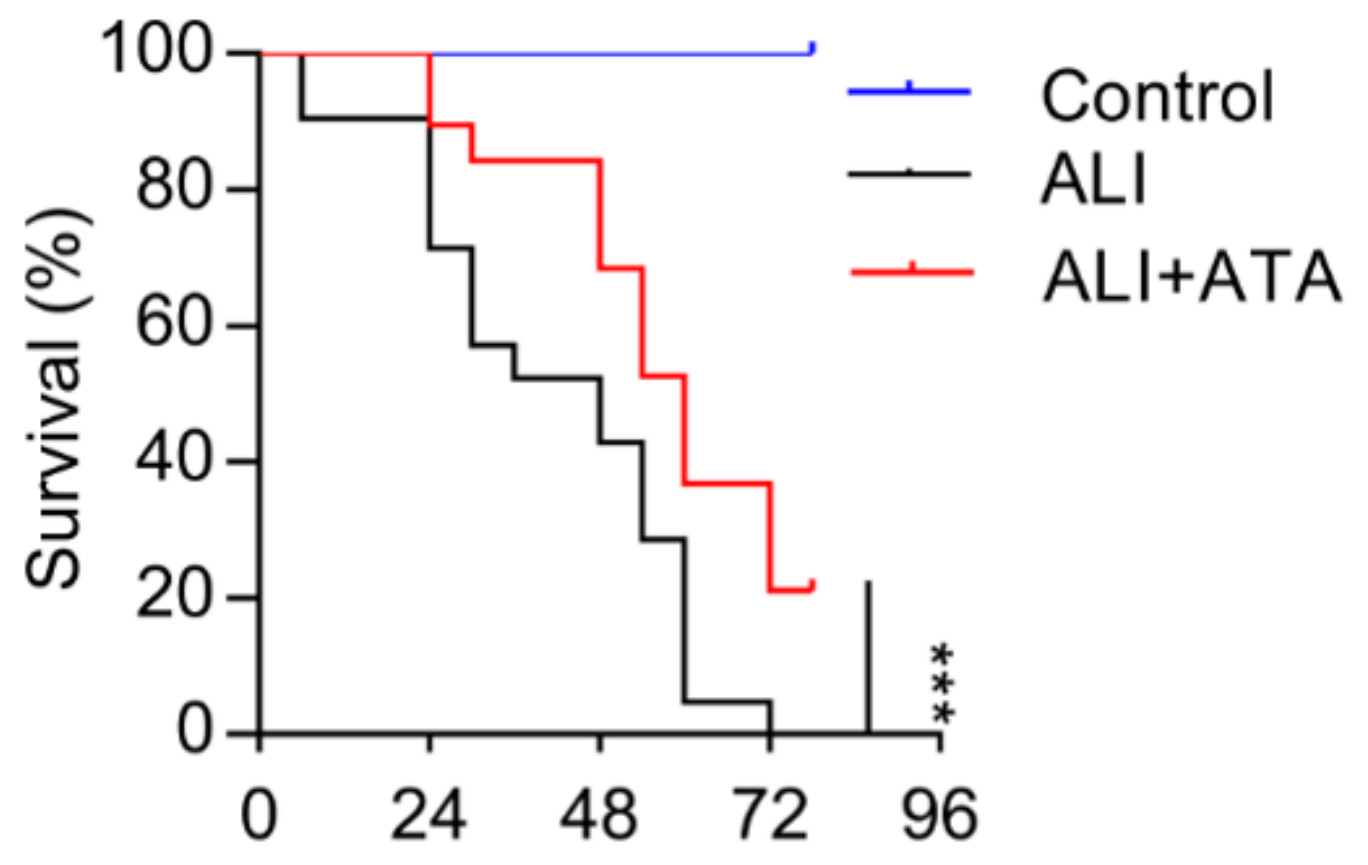

\section{Hours Post-LPS Administration}

\section{Figure 4}

Therapeutic blockade of Fn14 by ATA improves the survival of LPS-treated mice. ATA ( $20 \mathrm{mg} / \mathrm{kg})$ was administered to mice $2 \mathrm{~h}$ after induction of ALI by LPS $(25 \mathrm{mg} / \mathrm{kg})$. The mortality of the mice was monitored every $6 \mathrm{~h}$ over $96 \mathrm{~h}(\mathrm{n}=20)$. ${ }^{* \star *} \mathrm{P}<0.001$. 

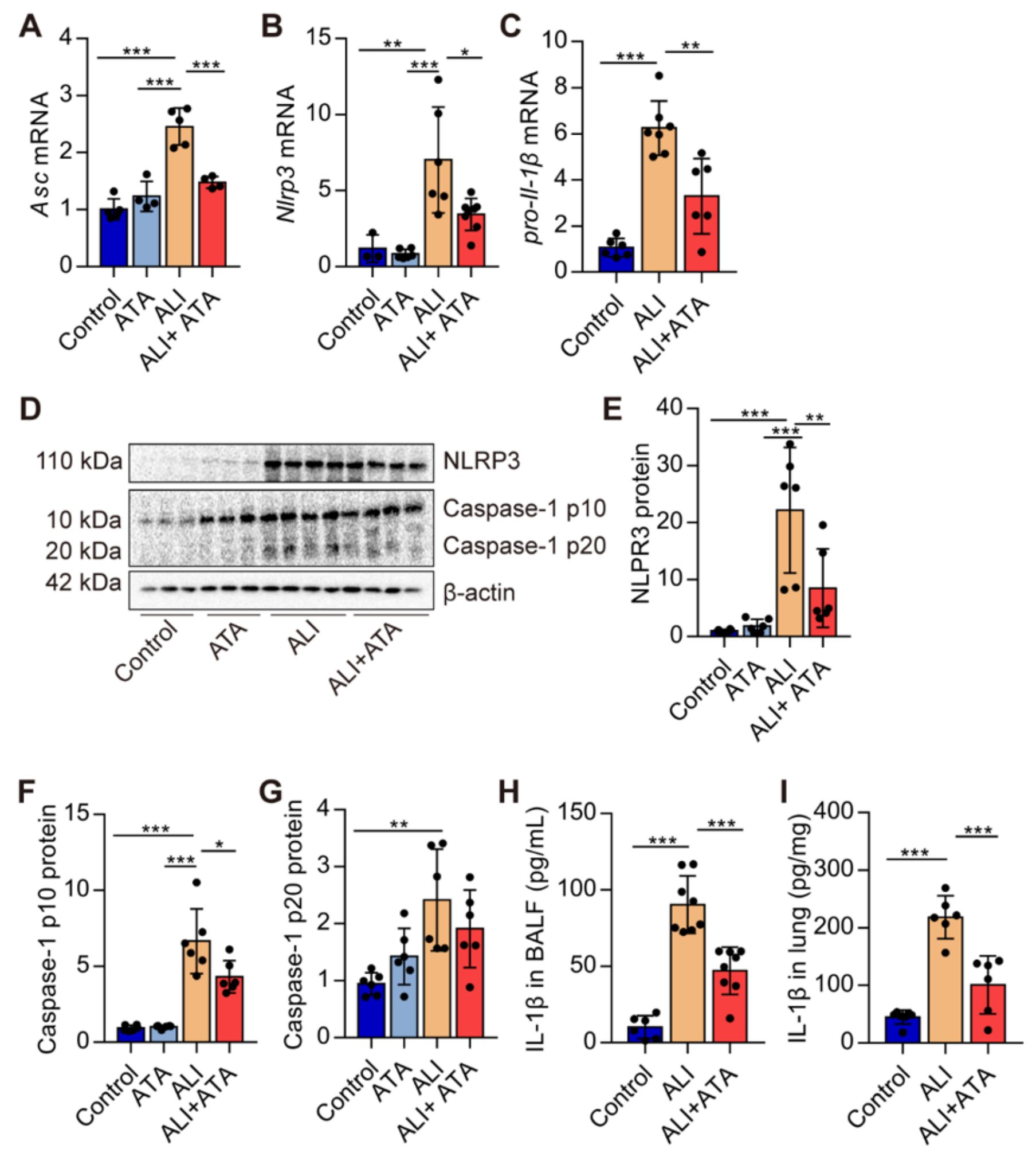

Figure 5

Blockade of Fn14 by ATA inhibits the NLRP3 inflammasome activation in ALI mice. Adult C57BL/6J mice were treated with saline or ATA (20 mg/ $\mathrm{kg})$. One hour later, mice were given saline or LPS $(5 \mathrm{mg} / \mathrm{kg}$, i.t.). (A-C) Twelve hours later, the expression of Asc, Nlrp3, and pro-II-1 $\beta$ mRNA in the lungs was determined by real-time PCR ( $n=3-7)$. (D-G) The levels of NLRP3, Caspase-1 p10, and Caspase-1 p20 protein in the 
lungs were determined by Western blot $(n=6)$. $(\mathrm{H}-\mathrm{I})$ The content of IL-1 $\beta$ protein in BALF and lung tissue was determined by ELISA $(n=6-8) .{ }^{*} P<0.05, * * P<0.01$, and $* \star * P<0.001$.
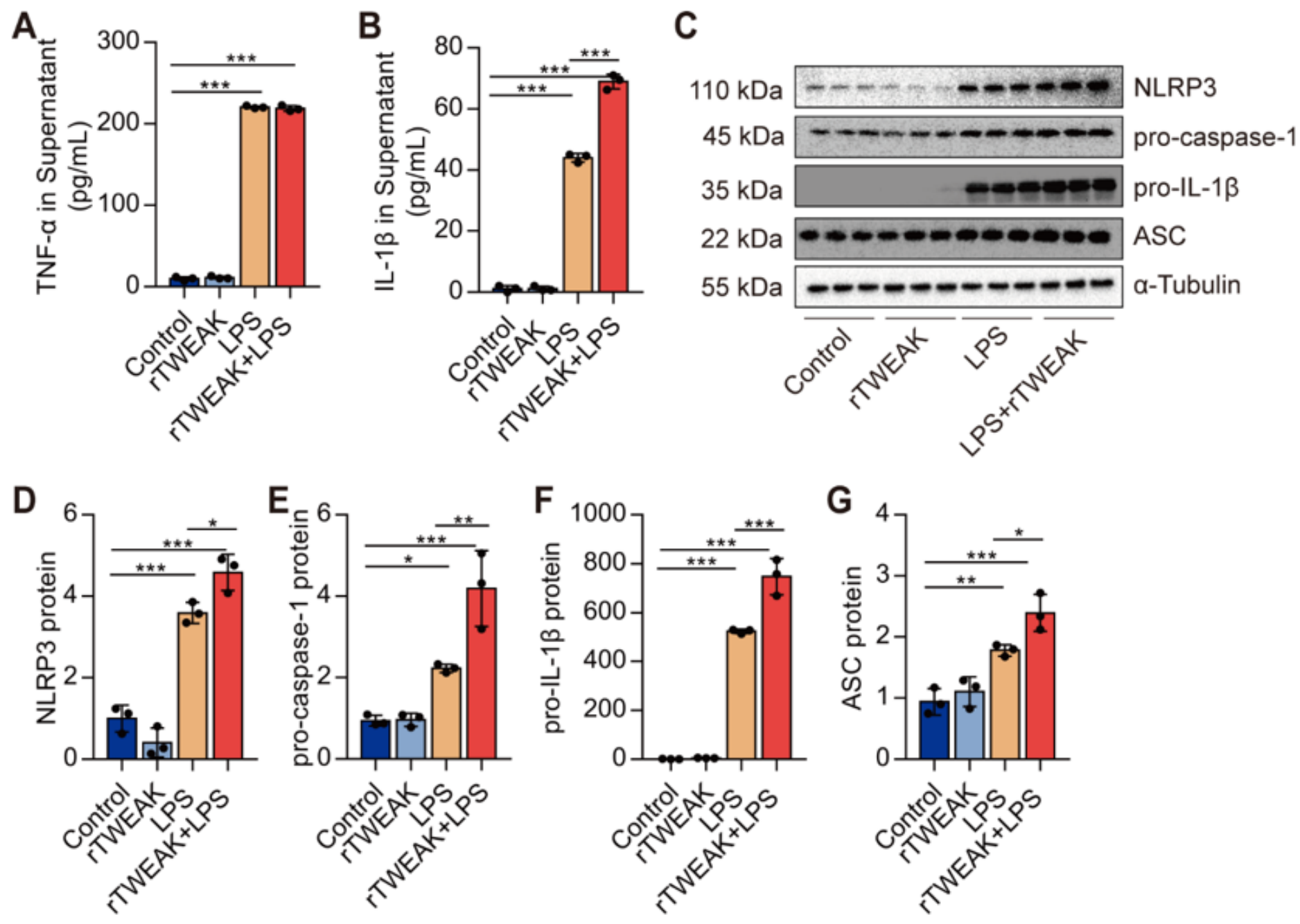

\section{Figure 6}

Activation of Fn14 by rTWEAK promotes the priming of NLRP3 inflammasome in macrophages. rTWEAK (100 ng/mL) was added to LPS (100 ng/mL)-challenged primary macrophages for $12 \mathrm{~h}$. (A-B) The contents of TNF- $a$ and IL-1 $\beta$ in the supernatant of primary macrophages were determined $(n=3)$. (C-G) The expressions of NLRP3, pro-caspase-1, pro-IL-1 $\beta$, and ASC protein in primary macrophages were determined by Western blot $(n=3)$. ${ }^{*} P<0.05, * * P<0.01$, and $* \star * P<0.001$. 

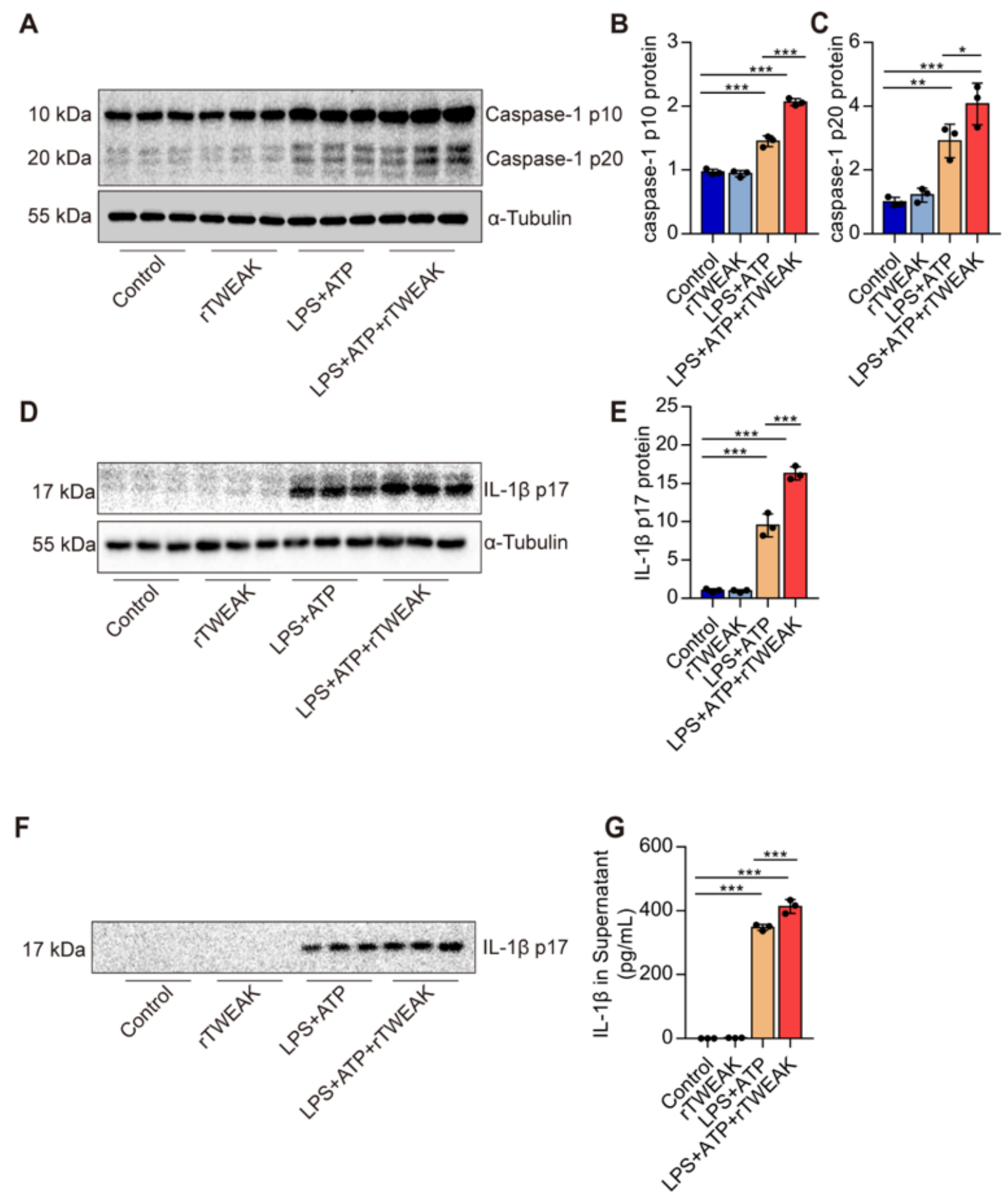

\section{Figure 7}

Pre-treatment of rTWEAK aggravates the activation of NLRP3 inflammasome in macrophages. The cells were stimulated by rTWEAK $(100 \mathrm{ng} / \mathrm{mL})$ and LPS $(100 \mathrm{ng} / \mathrm{mL})$ for $135 \mathrm{~min}$, and then ATP $(2.5 \mathrm{mM})$ challenged primary macrophages for $45 \mathrm{~min}$. (A-C) The levels of Caspase-1 p10 and Caspase-1 p20 protein in primary macrophages were determined by Western blot $(n=3)$. (D-E) The level of IL-1 $\beta$ p17 in primary macrophages was determined by Western blot $(n=3)$. (F) The level of IL-1 $\beta$ p17 in the 
supernatant of primary macrophages was determined by Western blot $(n=3)$. (G) The content of IL-1 $\beta$ p17 protein in the supernatant of primary macrophages was determined by ELISA $(n=3)$. ${ }^{*}<0.05, * \star P<$ 0.01 , and $* * * P<0.001$.
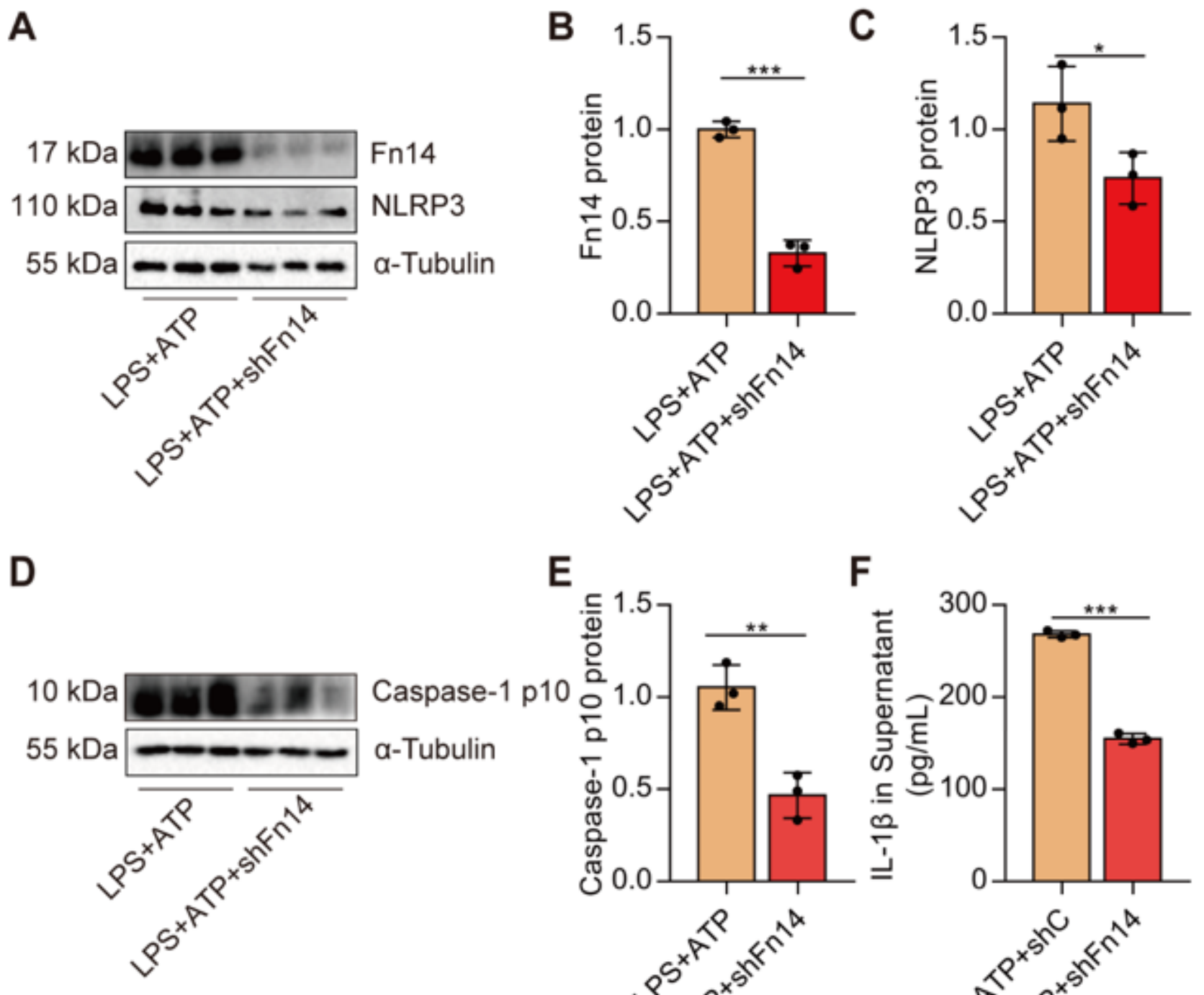

E

Caspase-1 p10
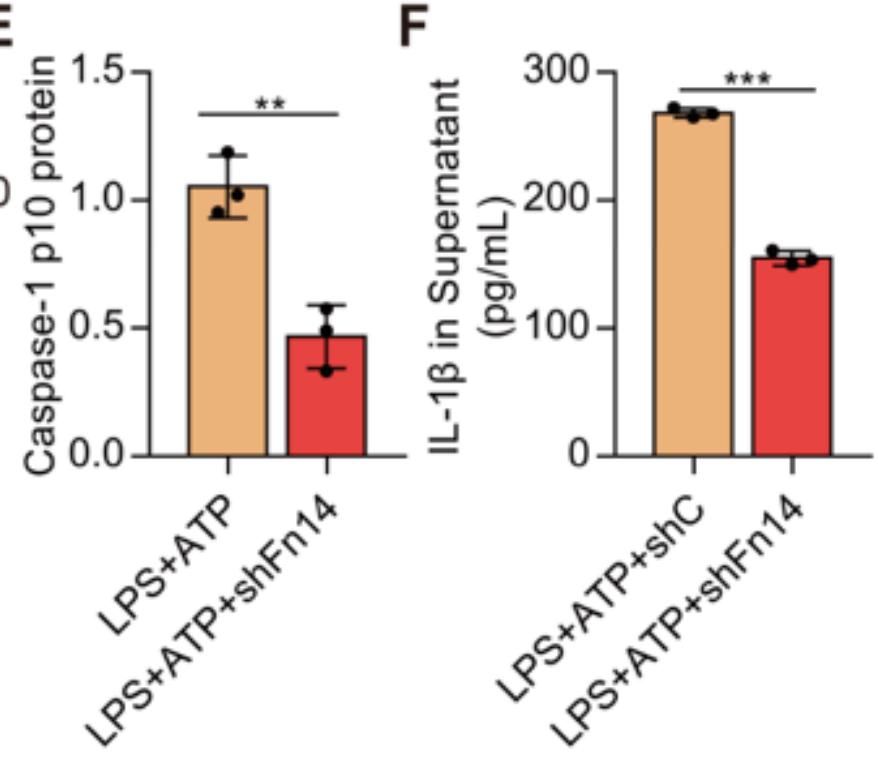

Figure 8

Silenced the expression of Fn14 blocks the activation of NLRP3 inflammasome in macrophages. Fn14 shRNA was added before LPS (100 ng/mL)-challenged primary macrophages for $96 \mathrm{~h}$. (A-C) The expressions of Fn14 and NLRP3 protein in primary macrophages were determined by Western blot $(\mathrm{n}=$ 3). (D-E) The level of Caspase-1 p10 protein in primary macrophages were determined by Western blot (n $=3)$. $(F)$ The contents of IL-1 $\beta$ in the supernatant of primary macrophages were determined $(n=3)$. ${ }^{2}<$ $0.05, * * \mathrm{P}<0.01$, and ${ }^{\star * \star} \mathrm{P}<0.001$. 

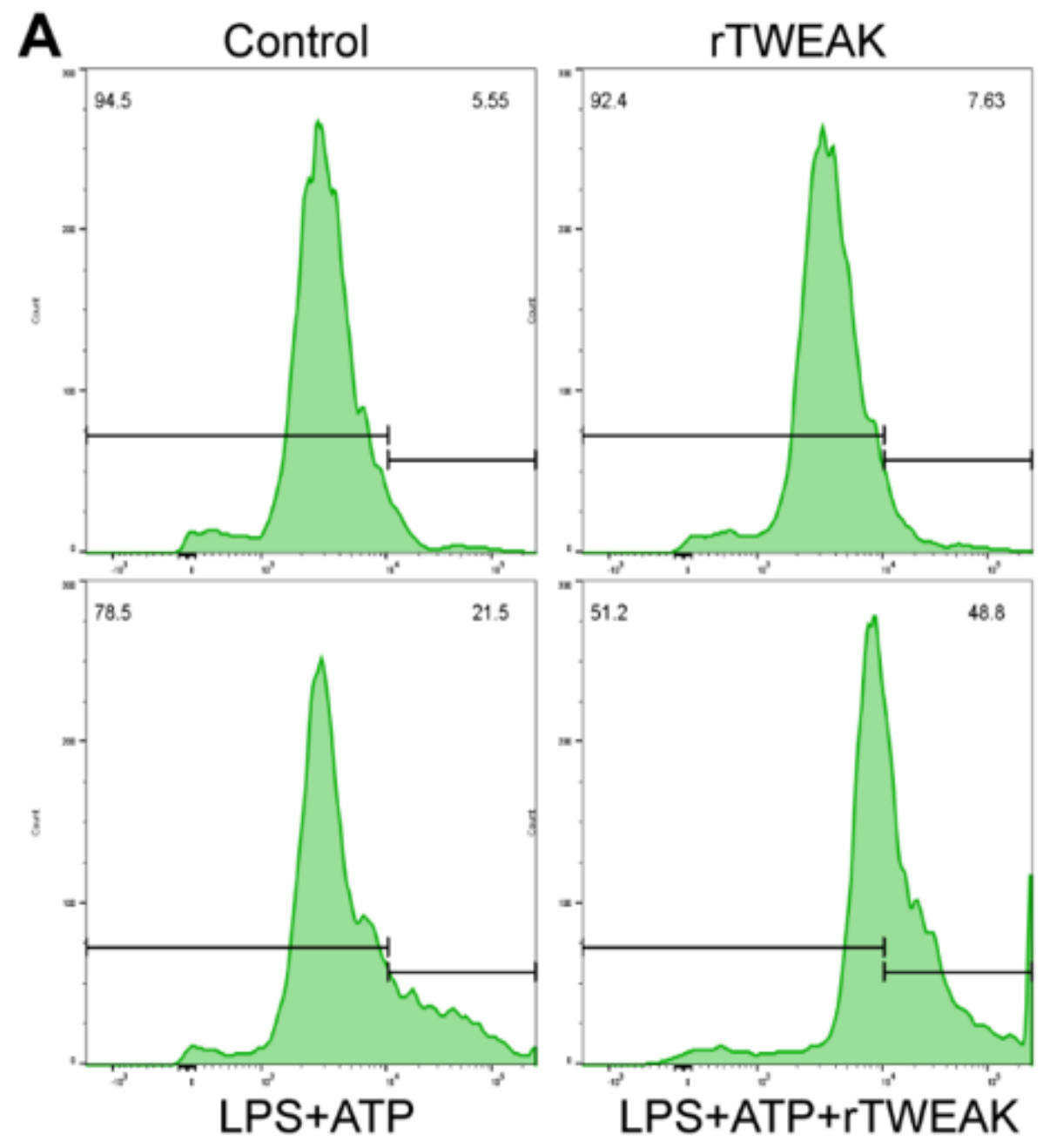

B

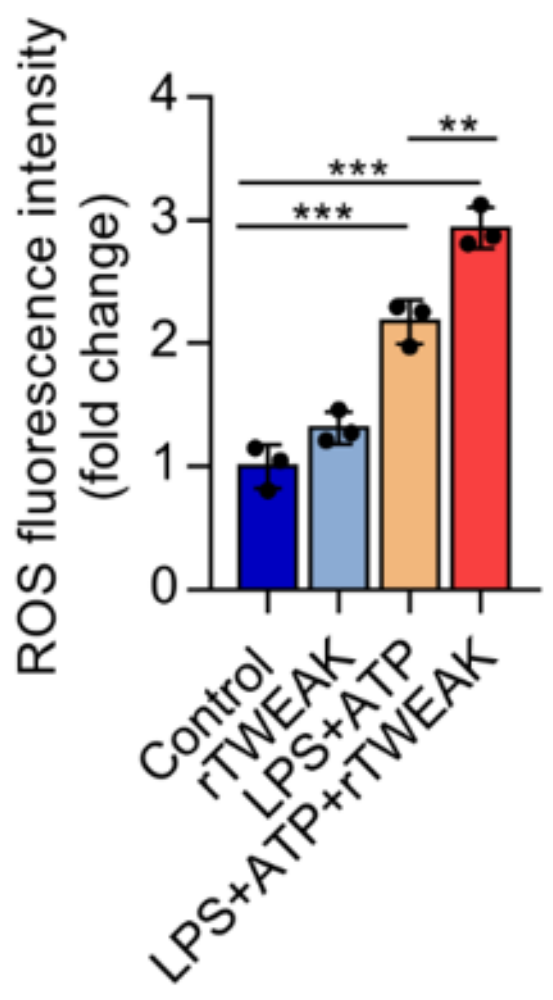

Figure 9

Pre-treated with rTWEAK aggravates the ROS production in macrophages. rTWEAK $(100 \mathrm{ng} / \mathrm{mL})$ was added to LPS (100 ng/mL) for $135 \mathrm{~min}$ and ATP $(2.5 \mathrm{mM}$ )-challenged primary macrophages for $45 \mathrm{~min}$. ROS production in the primary macrophages was analyzed by the flow cytometry assay $(n=3)$. ${ }^{*} P<$ 0.01 and $* * * P<0.001$. 